\title{
Alfabetización profesional de los estudiantes del Grado de Infantil a través de un Prácticum innovador
}

\section{Pre-Service Early Childhood Education Teachers' Professional Learning by Means of an Innovative Practicum}

\author{
Rosario Mérida Serrano ${ }^{1}$, María Elena González Alfaya ${ }^{2}$, María de los Ángeles \\ Olivares García ${ }^{3}$, Julia Rodríguez Carrillo ${ }^{4}$ \& Miguel Muñoz Moya ${ }^{5}$
}

Fecha de recepción: 12/10/2019; Fecha de revisión: 18/11/2019; Fecha de aceptación: 28/11/2019

Cómo citar este artículo:

Mérida-Serrano, R., González-Alfaya, M. E., Olivares-García, M. A., Rodríguez-Carrillo, J., \& MuñozMoya, M. (2019). Alfabetización profesional de los estudiantes del Grado de Infantil a través de un Prácticum innovador. Revista de Innovación y Buenas Prácticas Docentes, 8(4), 41-57.

Autor de Correspondencia: María Elena González Alfaya ed2goalm@uco.es

\section{Resumen:}

Se presenta la evaluación de una experiencia de innovación desarrollada en el Prácticum III de cuarto curso del Grado de Infantil de la Universidad de Córdoba, en el marco de la Red de Infantil EscuelaCentro de Profesorado-Universidad (RIECU). Se aplica el aprendizaje basado en proyectos (ABP) en las aulas infantiles. Participan 512 personas (niñas y niños de 3 a 6 años, familias, maestras y asesoras de Educación Infantil, alumnado de doctorado, estudiantes y profesorado universitario). Se utilizan tres estrategias de innovación: la pareja educativa, procesos de investigación-acción y procesos de documentación pedagógica. Se han utilizado dos instrumentos de recogida y producción de información: el grupo focal a las maestras y las memorias reflexivas de las estudiantes de Prácticum. Se realiza un análisis del discurso y se obtienen las siguientes conclusiones: Las estudiantes adquieren las siguientes competencias: (1) Comprender el valor de una planificación abierta y flexible; (2) Conocer ambientes de aprendizaje basados en la gestión colectiva del conocimiento; (3) Participar en situaciones de aprendizaje inclusivos que atienden a la diversidad; y (4) Comprender el valor de situaciones de aprendizaje en los que participa toda la comunidad, identificando los beneficios y debilidades de la participación familiar en los procesos educativos infantiles.

Palabras clave: Aprendizaje Basado en Proyectos, Diversidad, Educación Infantil, Formación Inicial Docente e Inclusión

\footnotetext{
I Universidad de Córdoba (España), ed1meser@uco.es; CÓDIGO ORCID: 0000-0001-9389-3263

2 Universidad de Córdoba (España), ed2goalm@uco.es; CÓDIGO ORCID: 0000-0001-8264-3680

${ }^{3}$ Universidad de Córdoba (España), ed1olgam@uco.es; CÓDIGO ORCID: 0000-0002-9440-5628

${ }^{4}$ Universidad de Córdoba (España), m12rocaj@uco.es; CÓDIGO ORCID: 0000-0003-0725-3054

${ }^{5}$ Universidad de Córdoba (España), p62mumom@uco.es; CÓDIGO ORCID: 0000-0001-6250-2995
} 

M.

\section{Abstract:}

This paper presents the assessment of an educational-innovation experience that has been carried out during the Practicum III of the 4th course of Early Childhood Education Degree (University of Córdoba), framed also in the RIECU (Early Childhood Education Schools-Center for Continuous Teacher TrainingUniversity) Network. The Project-Based Learning (PBL) method has been applied in Early Childhood classrooms. In sum, 512 people have been involved (3-6 years-old children, families, Early Childhood teachers and advisors, PhD students, pre-service teachers and University professors. Two different instruments for collecting and producing data have been used: a focus group with in-service teachers and reflective diaries written by the student-teachers from the Faculty of Educational Sciences. After carrying out a discourse analysis, the following final thoughts can be presented: Student-teachers have improved the following teaching competences: (1) To acknowledge the importance of an open and flexible planning, (2) To get to know learning environments that are based on a collective management of knowledge; (3) To participate in inclusive learning encounters aiming at attending to children's diversity; and (4) To understand the value of those learning situations which involve the entire community, and to identify the strengths and weaknesses of family participation in early childhood learning processes..

Key Words: Diversity, Early Childhood Education, Inclusion, Initial Teacher Training and Project-Based Learning 


\section{INTRODUCCIÓN}

La adquisición de una identidad docente ajustada es un proceso que se va adquiriendo paulatinamente. Hunde sus raíces en el desarrollo personal del futuro docente, así como en las experiencias vitales, académicas y profesionales en las que va participando (Castells, 2006). Sin embargo, teniendo poco en cuenta esta premisa de partida, la formación inicial docente que se imparte en las Facultades de Educación se sustenta en un plan formativo integrado por un conjunto de asignaturas que, de modo fragmentario y desde un enfoque sumativo, pretenden desarrollar las competencias que necesita un maestro o una maestra para desenvolverse eficazmente en los contextos educativos reales. Como consecuencia, se produce una incongruencia evidente entre la formación que reciben como estudiantes universitarios y las demandas que les exige una práctica profesional caracterizada por la incertidumbre, la simultaneidad y la imprevisibilidad, derivada de las interacciones sociales y los procesos de enseñanza que se producen en las aulas infantiles (Pérez Gómez, 2017).

La universidad ofrece un marco formativo caracterizado por enfoques de enseñanza eminentemente transmisivos, sustentados en enfoques teóricos muy alejados de la cotidianeidad y de los dilemas profesionales a los que han de enfrentarse día a día los docentes de la escuela actual (Crowther, 2001). De este modo, prevalecen procesos de enseñanza y aprendizaje centrados en el conocimiento declarativo y en la adquisición de estrategias y técnicas que responden más a una racionalidad técnica que a un enfoque práctico de la docencia (De Moya, Ferguson y Hann, 2009). Por ello, el alumnado universitario se va socializando paulatinamente en una cultura académica centrada en la adquisición de teorías bastante alejadas de su futura realidad profesional, aprendiendo que lo importante es memorizar unos contenidos que les permiten aprobar unas asignaturas para seguir avanzando en su desarrollo académico. A medida que cursan sus estudios de Grado interiorizan que hay una brecha o distancia enorme entre el decir (teoría) y el hacer (práctica) (Mérida, González y Olivares, 2012). Esta situación es lo que Korthagen (2010) denominó como la distancia que existe entre las teorías proclamadas y las teorías en uso, o dicho de otro modo, entre los principios educativos y el discurso políticamente correcto que los docentes suscriben y la realidad de sus prácticas y rutinas en el aula, las cuales en muchas ocasiones no se ajustan a la teoría conocida y expresada en las pruebas de evaluación que tienen lugar en la institución universitaria.

A esa perversa distancia entre el decir y el hacer profesional se une, en la mayoría de los casos, la omisión del desarrollo emocional que experimentan los futuros docentes en las aulas universitarias. Aún siguen vigentes prejuicios y estereotipos que identifican la educación emocional como un ámbito personal que ha de cultivarse y trabajarse fuera de la academia (Day, Kington, Stobart y Sammons, 2007). De esta manera, y siguiendo las directrices aportadas por Pérez Gómez (2017), en la formación docente inicial nos encontramos con la dificultad de construir un verdadero conocimiento práctico. Es decir, un conocimiento que corresponda a un proceso dialéctico de interacción permanente entre la teoría aprendida y los rasgos personales incorporados por cada individuo en el transcurso de su vida, la cual es fruto de la combinación de su personalidad y de las experiencias vitales que ha tenido. Por tanto, como formadores de maestros y maestras en la universidad hemos de propiciar situaciones de enseñanza-aprendizaje, donde los futuros docentes tengan oportunidad de confrontar la teoría con sus propios valores, experiencias y creencias, calando en 
su red emocional e intentando que transformen no solo su discurso teórico, sino sobre todo su compromiso con la práctica.

Porque la actividad docente es, ante todo, una acción relacional, comunicativa, contextual y ética (Perrenaud, 2007). Los futuros docentes deben entender que su posicionamiento ideológico es fundamental para posicionarse ante una realidad social que necesita ser analizada y transformada (Bauman, 2008). Estos profesionales han de asumir un compromiso ético con la mejora educativa, con la justicia social y con la concepción de la educación como herramienta de cambio. En definitiva, con la imbricación de la teoría, la práctica, los valores y las creencias personales de nuestro estudiantado estamos contribuyendo a transitar desde un conocimiento teórico educativo hasta un conocimiento profesional práctico (Sánchez, Pérez y Venegas, 2013). Un conocimiento práctico que sustenta un perfil de profesional docente reflexivo, que no solo ejecuta el currículo educativo en el aula, sino que también lo crea, recrea, analiza y adapta a las demandas contextuales, al hacer uso de su autonomía profesional.

Para conseguir el perfil de un maestro o una maestra como profesional reflexivo se necesitan, al menos, los siguientes requisitos (Mora, 2005): (1) Una teoría ajustada, útil y realista; (2) Una práctica apoyada en procesos de investigación-acción que favorezcan la reflexión en y sobre la acción y que permita el cuestionamiento de las ideas previas y creencias que el estudiantado posee sobre la docencia; (3) El aprendizaje por modelado y la interacción con referentes docentes de calidad; y (4) Conocer diseños y ambientes de aprendizaje centrados en el alumnado, donde el rol docente se descentraliza y se democratiza la adquisición del conocimiento.

\subsection{RIECU: un contexto para la alfabetización docente}

Con el fin de superar las debilidades expuestas en la formación inicial docente, desde el curso 2007/2008, se crea la Red de Infantil Escuela-Centro de ProfesoradoUniversidad (en adelante, RIECU). Se trata de una red profesional que, con el tiempo, se ha ido configurando como una comunidad de práctica, puesto que se sustenta en unos principios de funcionamiento horizontales, democráticos y dialógicos (Area, 2016). Esta red no reproduce la jerarquización y diferenciación habitual entre la teoría e investigación reservadas al profesorado universitario y la intervención y ejecución práctica en las aulas asignada a los maestros y las maestras. Por el contrario, es una red que se interesa por establecer puentes entre la formación inicial y continua, por conectar el saber novel y el saber experiencial, centrándose en las sinergias que se pueden establecer entre las nuevas generaciones de docentes y la experiencia de calidad acuñada tras años de formación (Mérida, González, Olivares y Rodríguez, 2018). El nexo entre las maestras de Infantil experimentadas y el alumnado universitario en la creación de esta red ha sido la aplicación del método de aprendizaje basado en proyectos (en adelante, ABP). Se trata de un método de aprendizaje que es abordado desde la formación universitaria y también desde la formación permanente en el Centro de Profesorado (en adelante, CEP). RIECU crea, de este modo, un espacio compartido en el que convergen los intereses del profesorado novel y el experto, constituyendo un contexto de reflexión sobre la formación docente, la adquisición de competencias profesionales y el modelo de educación para la infancia que deseamos.

RIECU, en estos 10 años de trayectoria, ha afianzado una línea de innovación docente consolidada, asentada en la colaboración, en la evaluación permanente de las experiencias que desarrollamos anualmente para acercar la teoría y la práctica, el saber novel y el saber experto, la formación docente inicial y continua (Olmedo, 2013). Se trata de una línea de innovación que no es estática, sino que avanza y se reformula año a año, porque las generaciones de jóvenes estudiantes de la Facultad de Ciencias 
de la Educación de la Universidad de Córdoba van cambiando, así como los pequeños y las pequeñas aprendices de las aulas infantiles y sus familias. Esta experiencia de innovación representa una oportunidad para consolidar las fortalezas, identificar las debilidades y proyectar sugerencias de mejora que nos van haciendo avanzar.

\subsection{El método de aprendizaje basado en proyectos: gestionar el conocimiento de la infancia}

Según Ruiz y Mérida (2016), los ABP son una iniciativa innovadora planteada por William H. Kilpatrick que empezaron a implantarse en el ámbito de la educación a partir de los años 80. Esta metodología, que tiene un gran potencial de desarrollo, actualmente no es utilizada de forma generalizada por los profesionales de la Educación Infantil (Balongo y Mérida, 2017; Imaz, 2015). En este marco metodológico se ponen en marcha procesos de investigación donde los niños y las niñas se relacionan, exploran, indagan e investigan su entorno más cercano, dando respuesta a su curiosidad innata y a su incansable deseo de conocer. Precisamente, en el aula donde se trabaja el ABP se respeta la forma natural de los pequeños y las pequeñas, utilizando el juego, el lenguaje, la interacción social, la observación y la motivación como resortes para que el aprendizaje sea efectivo y duradero en el tiempo (Balongo y Mérida, 2017).

Se trata de un modelo educativo que responde a una concepción socioconstructivista del aprendizaje (Gómez y Pérez, 2011) y que se apoya en los siguientes principios educativos:

(1) Respeto y escucha a la infancia, valorando que los niños y las niñas no son recipientes que hay que llenar, sino agentes activos de aprendizaje que poseen su propia cultura (Busquet-Duran, Medina-Cambrón y Ballano-Macías, 2013), que construyen, crean y recrean la información hasta convertirla en conocimiento.

(2) Fomento de las inteligencias múltiples (Gardner, 2008, 2010) porque los niños y las niñas poseen diferentes habilidades y destrezas que hay que descubrir, valorar y potenciar. Cada aprendiz tiene un talento, una capacidad especial, una competencia que lo singulariza y diferencia del resto. A través del ABP se crean ambientes de aprendizaje estimulantes, variados, diversos, en los que cada aprendiz puede encontrar su espacio, desarrollar su pasión y proyectar su creatividad. No son ambientes uniformantes ni cerrados, donde todo el alumnado ha de realizar la misma tarea en el mismo tiempo, sino que por el contrario las propuestas y situaciones de aprendizaje son abiertas, flexibles y, en buena medida, imprevisibles (Pegalajar, 2018).

(3) Construcción de un conocimiento relevante y significativo, centrado en la conexión sustantiva entre las ideas y experiencias previas del alumnado y las nuevas propuestas de aprendizaje. Un conocimiento que surge fruto de la interacción dialéctica entre los esquemas de conocimiento que poseen los aprendices y las reestructuraciones que generan las nuevas experiencias de aprendizaje, las cuales responden a sus intereses y necesidades vitales (Escribano y Martínez, 2013). Por tanto, es un aprendizaje que trasciende las paredes de la escuela, que supera los estrechos límites de la escolaridad y que se transfiere a la vida cotidiana, al ser un conocimiento útil para desenvolverse 
eficazmente en el entorno cotidiano (Hughes, Hughes y Hodgkinson, 2016). Es un conocimiento que sirve para situarse adecuadamente en el mundo, para comprender la realidad que les rodea, para cuestionarse el contexto físico y social, y para posicionarse como agente activo en su transformación.

(4) Un enfoque de investigación, en el que el conocimiento no solo se memoriza y se reproduce, sino que se construye en un proceso de exploración y búsqueda permanente, asemejándose al procedimiento científico. El alumnado parte de unas hipótesis iniciales y provisionales que han de verificar o refutar con evidencias empíricas extraídas a base de observaciones, exploraciones, comparaciones, análisis, clasificaciones, asociaciones, etc. (De Moya y Madrid, 2015).

(5) Un enfoque comunitario del saber, en el que no solo sabe y enseña el docente, sino que existe una comunidad de aprendizaje donde los saberes se construyen en procesos colectivos multidireccionales y los roles de enseñante y aprendiz se diluyen y se comparten (Zapata, 2015). Se crean ecosistemas de aprendizaje colectivo en los que, aunque el liderazgo de los procesos educativos los detenta el o la docente, se valora, incentiva y facilita la aportación de las familias. Es una manera de aprovechar el conectivismo para fomentar el aprendizaje complejo que se produce en un mundo social digital en rápida evolución.

(6) Un enfoque complejo y globalizado del conocimiento que supera el fraccionamiento del saber al separar el aprendizaje por bloques o asignaturas, proponiéndose por el contrario un planteamiento de aprendizaje más flexible, sin rigidez, centrado en las asociaciones y múltiples conexiones que se establecen entre los nodos informacionales y las redes, las cuales generan el aprendizaje (Cabello, 2011). Como se señala en el Informe The Learning Society (2010), los sistemas educativos necesitan pasar a convertirse en una sociedad del aprendizaje.

\subsection{Educar a los nativos digitales: un nuevo rol docente en la era digital}

Como se ha señalado anteriormente, el aprendizaje en la era digital se ha modificado sensiblemente respecto a momentos históricos anteriores (Wake, Dysthe y Mjelstad, 2007), en los que el formato predominante de enseñanza-aprendizaje era liderado por el o la docente, en un posicionamiento directivo y unilateral. Como consecuencia de este cambio es necesario modificar la forma de enseñar, teniendo en cuenta las necesidades de nuestros pequeños nativos interactivos digitales (INTEF, 2014; Prensky, 2011).

Según Viñals y Cuenca (2010):

Los denominadores más comunes que se atribuyen al nuevo rol del docente de la era 2.0 son: organizador, guía, generador, acompañante, coacher, gestor del aprendizaje, orientador, facilitador, tutor, dinamizador o asesor. Estos nuevos roles se asientan en la idea de cambiar la transmisión unidireccional del conocimiento por el intercambio horizontal de información, abundante, caótico y desestructurado (p. 110).

En definitiva, y siguiendo las sugerencias mostradas por Bates (2015) en su último libro Teaching in a Digital Age, los maestros y las maestras en la era digital deben adoptar una actitud de indagación permanente, generar entornos de aprendizaje estimulantes, desarrollar el dominio de competencias, atender a las singularidades de cada aprendiz, ajustando la enseñanza a las demandas 
diferenciadas de cada uno de ellos y ellas, potenciar el trabajo colaborativo y desarrollar un espíritu ético. La tecnología y la información por sí solas no guían ni aconsejan al alumnado; por ello, la labor del docente en la educación digital es hoy más importante que nunca.

Entonces, ¿cómo podemos desarrollar estas competencias docentes necesarias para los futuros maestros y las futuras maestras? Partiendo de que el entorno de formación universitaria, sustentado en un plan de estudios articulado en asignaturas entendidas como "compartimentos estancos" no es el mejor contexto para el desarrollo de competencias, nos centramos en el Prácticum como un espacio de aprendizaje profesional que reúne unas características privilegiadas para potenciar la asunción de nuevos roles docentes que den respuesta a las necesidades de la era digital (Capraro, Capraro y Helfeldt, 2010).

Pero no cualquier experiencia de prácticas en los centros genera directamente una cualificación profesional para la era digital como la que deseamos. Los aprendizajes adquiridos por nuestros estudiantes dependerán mucho de la calidad del docente al que acompañen, de su actitud en el aula y de los ambientes de aprendizaje que cree. Diversos estudios e investigaciones previas (Mérida, González, Olivares y Rodríguez, 2018), muestran que hay que diseñar un prácticum de calidad para incrementar las competencias profesionales de los futuros docentes. El prácticum RIECU es una innovación educativa que pretende favorecer el dominio de las competencias profesionales docentes de los estudiantes del último curso del Grado de Educación Infantil.

\section{DESARROLLO DE LA EXPERIENCIA DE INNOVACIÓN}

\subsection{Objetivos}

Los principales propósitos que se plantean en la innovación del Prácticum RIECU son:

- Propiciar el encuentro entre maestras seleccionadas por su excelencia docente y estudiantes de Magisterio.

- Ofrecer a nuestros estudiantes unos modelos docentes excelentes, caracterizados por su compromiso con la formación permanente, la innovación y la investigación educativa.

- Poner en marcha procesos de investigación-acción colaborativa compartidos entre las maestras, las asesoras de Infantil y las estudiantes y profesoras universitarias.

- Socializar a las estudiantes de Magisterio en una cultural docente en Infantil centrada en la metodología ABP.

- Intensificar la colaboración entre la formación docente inicial y la continua.

- Mejorar la comunicación, valores y rutinas creadas entre quienes participan en la comunidad de práctica RIECU (Red de Infantil Escuela-Centro de profesoradoUniversidad) 
- Orientar al alumnado universitario en la construcción de su proyecto profesional a partir de los modelos docentes de excelencia con los que ha trabajado en su trayectoria formativa inicial.

\subsection{Fases de la innovación}

Las fases en las que se articula esta innovación son:

(1) Planificación. Mediante dos encuentros de la Red RIECU se acuerdan: a) El número de estudiantes de Magisterio que va a participar; b) El número de maestras y maestros que van a colaborar en su tutela; c) Los procesos formativos sobre ABP que se van a desarrollar en la Facultad de Educación y en el CEP, y d) La documentación y orientaciones para el desarrollo de los proyectos que se van a abordar en las aulas infantiles.

(2) Desarrollo. Tienen lugar tres momentos claves en esta fase: a) La presentación pública del proyecto de innovación, donde se forman las parejas educativas; b) El desarrollo de los procesos de investigación-acción durante las prácticas en los centros de Educación Infantil; y c) La elaboración de los procesos de documentación pedagógica, donde se recogen evidencias empíricas (fotografías, producciones infantiles y vídeos) sobre el desarrollo de los proyectos.

(3) Evaluación. El proyecto de innovación se evalúa de dos formas: a) Las profesoras y maestras participantes realizan la evaluación a través de un Grupo de Discusión; b) Las estudiantes de Magisterio realizan su valoración en su memoria reflexiva de prácticas.

\subsection{Participantes}

Han participado las siguientes personas:

Tabla 1.

Participantes en la experiencia.

\begin{tabular}{lc}
\hline \multicolumn{1}{c}{ Colectivo } & Cantidad \\
\hline Niños y niñas de 3 a 6 años & 438 \\
\hline Maestras de $2^{\circ}$ ciclo de Ed. Infantil & 22 \\
\hline Estudiantes del Grado de Infantil & 22 \\
\hline Profesoras universitarias & 3 \\
\hline Estudiantes de doctorado & 2 \\
\hline Asesoras de Infantil del CEP & 2 \\
\hline Familias & 23 \\
\hline TOTAL & 512 \\
\hline
\end{tabular}

Fuente: Elaboración propia

\subsection{Contexto}

Esta experiencia de innovación se desarrolla en el marco del Prácticum III, que se ubica en cuarto curso del Grado de Educación Infantil de la Facultad de Ciencias de la Educación de la Universidad de Córdoba. Participan, de forma voluntaria, 22 estudiantes, las cuales son tutorizadas por 22 maestras seleccionadas por el Centro de Profesorado Córdoba "Luisa Revuelta", en función de los siguientes criterios: (1) Formación en ABP; (2) Experiencia de aplicación real de la metodología de proyectos en las aulas infantiles; (3) Compromiso para desarrollar procesos de investigaciónacción; y (4) Participación en el grupo focal para evaluar la experiencia. 
El Prácticum III se articula de la siguiente manera: (1) Seminarios de preparación, donde se explica el marco normativo de Prácticum diseñado por la Comisión de Prácticum de la Facultad y se asignan los centros elegidos por el alumnado universitario; (2) Prácticas presenciales. Durante dos meses (marzo-mayo) el alumnado asiste al centro y desarrolla procesos de investigación-acción; (3) Seminarios de análisis. Se dan orientaciones para la elaboración de la memoria reflexiva, se comparten las experiencias vividas en los centros y se evalúa la experiencia de innovación.

\subsection{Interrogantes de investigación}

- ¿Qué aprendizaje sobre la planificación docente adquiere el estudiantado al desarrollar el ABP en el prácticum innovador RIECU?

- ¿Qué aprendizaje sobre la convivencia y la interacción social del aula adquiere el estudiantado al desarrollar el ABP en el prácticum innovador RIECU?

- ¿Qué aprendizaje sobre la atención a la diversidad adquiere el estudiantado al desarrollar el ABP en el prácticum innovador RIECU?

- ¿Qué aprendizaje sobre la participación de la comunidad adquiere el estudiantado al desarrollar el ABP en el prácticum innovador RIECU?

\subsection{Instrumentos de recogida y producción de la información}

Se han utilizado dos tipos de datos para evaluar esta experiencia de innovación: a) Textuales, recogidos a través de las memorias reflexivas de las estudiantes del Grado de Infantil y de la transcripción del Grupo Focal organizado con las maestras de Educación Infantil; b) Visuales, incluidos en la documentación pedagógica (imágenes, fotos, vídeos y producciones infantiles) realizadas por las estudiantes universitarias.

\subsection{Técnicas de análisis de datos}

Se ha usado la técnica de análisis del discurso, para interpretar los significados incluidos en la narración de las maestras y estudiantes de Educación. El análisis del discurso se llevó a cabo identificando dos tipos de unidades de análisis: (1) Dimensiones, relacionadas con los objetivos y que derivan de la teoría suscrita, son de carácter macro; (2) Categorías, que emergen de los datos y tienen un carácter más micro. Dichas categorías se han codificado y se han clasificado respetando un acuerdo interjueces, en las que se han desestimado las discrepantes y se han asumido las coincidentes. La codificación corresponde a: (1) Inicial del instrumento: M-Memoria o GF-Grupo Focal; (2) Inicial del colectivo: A-Alumna ó M-Maestra; (3) Número. Para anonimizar la identidad de las participantes se las identifica con un número. De esta manera, por ejemplo, MA18 significa que el discurso ha sido extraído de la Memoria de la alumna 18 y GFM13 significa que es una intervención realizada en el Grupo Focal por la maestra a la que se le ha asignado el número 13.

La triangulación de instrumentos (memorias reflexivas y grupo focal) y la triangulación entre informantes (maestras y estudiantes universitarias), permite minimizar sesgos e incrementar la credibilidad y consistencia de este trabajo (Cisterna, 2005). 


\section{RESULTADOS}

Para analizar los datos se ha elaborado una matriz hermenéutica que muestra la relación entre las distintas unidades de análisis -dimensiones y categorías- utilizadas:

Tabla 2.

Matriz hermenéutica

\begin{tabular}{ll}
\hline \multicolumn{1}{c}{ Dimensiones } & \multicolumn{1}{c}{ Categorías } \\
\hline 1. Planificación didáctica & $\begin{array}{l}\text { 1.1. Improvisación o planificación abierta } \\
\text { 1.2. Intereses del alumnado versus planificación cerrada }\end{array}$ \\
& $\begin{array}{l}\text { 1.3. De la planificación a la creación de ambientes de } \\
\text { aprendizaje }\end{array}$ \\
\hline 2. Convivencia & $\begin{array}{l}\text { 2.1. Gemocratización del aprendizaje } \\
\text { 2.2. Comunidad de práctica }\end{array}$ \\
\hline 3. Atención a la diversidad & $\begin{array}{l}\text { 3.1. Ayuda mutua } \\
\text { 3.2. Inclusión de las diferencias }\end{array}$ \\
\hline 4. Apertura a la comunidad & 4.1. Inteligencia distribuida \\
\hline & 4.2. Conectivismo y participación social \\
\hline
\end{tabular}

Fuente: Elaboración propia.

La primera dimensión relacionada con la planificación didáctica las estudiantes de Ciencias de la Educación manifiestan, por amplia mayoría (86\%), que al inicio de sus prácticas pensaban que la metodología $A B P$ era un método en el que se improvisaba mucho. Sentían bastante inseguridad al no llevar preparadas las fichas y propuestas de aprendizaje al aula. No entendían el concepto de planificación abierta, y echaban de menos, para sentirse seguras, tener un guion claramente delimitado de lo que iban a hacer en el aula durante toda la jornada.

"Yo estaba muy nerviosa, es como si vas a clase a ver qué se les ocurre cada día a los niños..." (MA4).

"Es que llegar sin nada así concreto, yo que sé, como una ficha o un juego... era muy fuerte... Yo temía que los niños no dijeran nada y entonces no saber qué hacer" (MA18).

También las maestras indican que las estudiantes de Ciencias de la Educación cuando llegan al aula tienen una dificultad para escuchar y respetar los intereses de los niños y las niñas, y apuestan por una planificación cerrada.

"Marta quería ir por delante de los niños... Prepararlo todo, tenerlo todo controlado... Le agradezco su preocupación, pero sus nervios le impedían escuchar lo que decían los niños" (GFM13).

"Mi práctica quería conocerlo todo de antemano... Luego se ha ido relajando, pero al principio quería llevarlo todo cerrado y programado antes... Yo le decía, pero chiquilla déjalos que se expresen... Tú síguelos, ellos te indican el camino...".

Pese a la actitud inicial de intervención y control por parte de las estudiantes universitarias que relatan las maestras, la tendencia de más de la mitad de las docentes es reconocer que este posicionamiento inicial de las alumnas de Grado ha ido evolucionando a medida que transcurría su estancia en las aulas. Han ido evolucionando desde una preocupación por tener una planificación cerrada que dirigiera sus actuaciones y tranquilizara sus incertidumbres, hacia un interés creciente por crear ambientes de aprendizaje motivadores. Han transitado desde la 
preocupación por el control del aula hacia la preocupación por generar situaciones de aprendizaje relevantes y con sentido.

"Sí, sí... la mía (se refiere a la alumna de prácticas) primero estaba agobiada por tenerlo todo programado, luego ya se metió en el proyecto y se interesaba por estimular a los niños, por buscar materiales..." (GFM17).

Son las propias alumnas las que manifiestan en sus memorias que han entendido y experimentado eso que tanto se repite durante la formación universitaria en diversas materias (Sistema Educativo, Planificación e Innovación y Estrategias de Intervención en Educación Infantil): el respeto y la escucha a los niños y las niñas. Admiten que, a nivel teórico, es muy fácil suscribir este principio, pero que en la práctica resulta muy complicado aprender a ser maestra "de otra manera". Ellas lo explican del siguiente modo:

"Es que claro... después de tanto tiempo... tú cuando vas al aula no sabes actuar de otra manera... Es la imagen habitual de una maestra... enseñar y que los niños la escuchen lo que les dice..." (MA12).

"A mí me daba coraje... porque cuando menos acordaba estaba yo enseñando lo que yo sabía pero que no me habían preguntado y probablemente no les interesara... Los niños se merecen que se les escuche, debemos aprender a democratizar su aprendizaje... Debemos desaprender la imagen de maestra que tenemos en la cabeza" (MA6).

La segunda dimensión incluida en la matriz hermenéutica está referida a la convivencia. Un porcentaje considerable $(73 \%)$ de alumnas indica que uno de los pilares básicos del ABP es la gestión colectiva del conocimiento. Aluden a la estructura cooperativa que perciben en el aula, lo cual contrasta con otras experiencias vividas en Prácticums anteriores, en las que podían apreciar una estructura individualista y competitiva del aprendizaje. Sin embargo, llama la atención que la gestión cooperativa del aula no es valorada positivamente por una parte significativa de las estudiantes (32 $\%$ :

"Yo creo que está bien lo de ponerlos en grupo, pero lo malo es que se copiaban unos de otros..." (MA2).

"Los que saben menos como están todos juntos siempre se fijan de los más espabilados..." (MA1).

La percepción de las maestras en esta dimensión es discrepante respecto a las alumnas. Un total del $73 \%$ de las maestras indican que una de las fortalezas fundamentales del ABP es la posibilidad de que el aula se configure como una comunidad de práctica. Las maestras consideran que la colaboración y la interacción social entre iguales es una herramienta didáctica de primer orden para el aprendizaje. Se percibe un posicionamiento en la teoría sociocultural, al considerar que el desarrollo personal es una construcción cultural que se realiza a través de la interacción con otras personas de una determinada cultura mediante la realización de actividades sociales compartidas (Vygotsky, 1989). Siguiendo los planteamientos vygotskianos, las docentes defienden que en el desarrollo cultural del niño toda función aparece dos veces: primero a nivel social y más tarde, a nivel individual; 
primero entre personas (interpsicológica), y después, en el interior del propio niño (intrapsicológica):

"Con los proyectos los niños aprenden por grupos, cada uno aporta una idea, unos aprenden de otros y los más rezagados imitan a los más avanzados" (GFM5).

"La convivencia en el aula es fundamental, no solo para el comportamiento social, sino sobre todo para el aprendizaje. Se crea con los proyectos como una comunidad de práctica, cada uno hace lo que mejor se le da y ayuda a quien tiene dificultades, de forma natural..." (GFM7)

El clima de convivencia que se genera en el ABP es de distensión, de seguridad, de colaboración y de ayuda. Se entiende que el objetivo último a conseguir se alcanza si participan todos los compañeros y las compañeras, y si se instaura un principio de funcionamiento de ayuda mutua entre iguales. Esta percepción es compartida por docentes y estudiantes:

"Sí ellos se ayudan de forma natural, yo no les digo nada...Les sale espontáneo, es un hábito creado en clase...es una ayuda recíproca que les brota..." (GFM8).

"Me quedé sorprendida cuando veía que los niños se ayudaban entre sí, sin tener que decirles nada. Era muy diferente a lo que he visto otros años en las prácticas..." (MA11).

La dimensión que alude a la atención a la diversidad en el aula integra dos categorías fundamentales: la que se refiere a la personalización del proceso de aprendizaje y la que hace alusión a la inclusión de las diferencias. Comprobamos que las maestras tienen un concepto de diversidad amplio, que no se reduce a la identificación de diversidad con discapacidad. Por su parte las estudiantes presentan, en su mayoría, una visión de diversidad más sesgada.

"Claro, cada niño es un mundo... la diversidad se ve en muchas cosas... en los intereses, las motivaciones, el ritmo de aprendizaje... depende mucho de los niños y de sus familias..." (GFM14).

"La diversidad se atiende con el ABP... había dos niños con TDH en el aula y se integraban muy bien en el proyecto..." (MA15).

Tanto las maestras como las alumnas consideran que atender la diversidad del aula supone desarrollar procesos educativos ajustados a las necesidades personales de cada aprendiz. El acompañamiento educativo de la maestra, la dificultad de las tareas propuestas, la aceptación de diferentes niveles de ejecución de las actividades, el planteamiento de situaciones de enseñanza-aprendizaje abiertas, la propuesta de ambientes de aprendizaje globalizados, en los que se integran tareas que requieren el uso de las inteligencias múltiples, el diseño de propuestas de aprendizaje individualizadas, la ayuda entre iguales y las actividades en grupos heterogéneos, son algunas de las medidas que, en opinión de las docentes y de las estudiantes, ayudan a respetar las diferencias del alumnado.

"Todos los niños se sentían muy bien... Ninguno decía yo no sé hacer esto o no puedo... Cada uno lo hacía según sus posibilidades" (MA19).

"Al final yo prefería que Gustavo (un niño con TDAH) no saliera del aula... lo veía mejor atendido en la clase... más centrado, a ver tenía días... pero igual que en la clase de PT... sus compañeros siempre le ayudaban y él no se 
quería ir cuando hacíamos el proyecto. Era superexperto en los castillos medievales... jejeje" (GFM4).

La última dimensión está referida a la apertura a la comunidad. Las maestras asumen que el conocimiento en la era digital no solo reside en el docente. Por el contrario, defienden una concepción de inteligencia distribuida, al considerar que cada persona tiene un saber que puede aportar, creándose redes y nodos informacionales en las que el conocimiento se democratiza y fluye de manera multidireccional entre las diferentes personas.

"Al final, lo bonito, es que todos aprendemos de todos... No solo sé yo como maestra... es más yo no sé de muchas cosas... y podemos acudir a otros profesionales, instituciones y san internet (risas) para construir conocimiento..." (GFM20).

Por su parte las alumnas tienen claro que el conectivismo es la teoría de aprendizaje que prevalece en la era digital. Las TIC y las redes sociales inciden en los niños y las niñas que, como nativos digitales, aprenden de manera multidireccional y no solo de las explicaciones de la maestra. Consideran este modelo obsoleto y apuestan por un conocimiento dialógico y abierto a la participación social.

"Yo lo he visto claro, no solo soy yo como maestra la que tengo que enseñar... la comunidad o la tribu educa... yo tengo que aprender mucho con y de ellos... Ahora todo el mundo sabe de todo lo que le interesa... Internet te lo facilita..." (MA22).

Para concluir, las maestras consideran a las familias como agentes de colaboración y apoyo para desarrollar procesos educativos que trascienden las paredes del aula, las visualizan como personas interesadas en el fomento y apoyo de la educación de sus hijos e hijas, y que están, en la mayoría de los casos, dispuestas a colaborar. Las estudiantes no siempre consideran que la participación familiar sea una estrategia adecuada, percibiendo disfunciones en el comportamiento de alguna de ellas y reivindicando una autonomía profesional frente a las mismas.

"Yo creo que las familias son fundamentales, sin ellas no podríamos desarrollar el ABP... Casi siempre se entregan a tope..." (GFM21).

"Considero acertada la colaboración familiar, pero no siempre saben colaborar bien. Había una madre en clase que interfería en las decisiones de la maestra y yo considero que quien debe decir lo que debe hacer el niño en la clase es la maestra" (MA22).

"Que las familias colaboren está bien. Pero hay algunas que no salen de la clase y me parece demasiado" (MA21).

"Alguna madre intervenía en cosas que no debería, como por ejemplo donde se sienta su hijo" (MA13).

"La madre venía de excursión, pero solo se ocupaba de su hija. Eso no me parece bien" (MA18). 


\section{CONCLUSIONES}

La evaluación de la experiencia de innovación en el Prácticum RIECU nos permite dar respuesta a los interrogantes de investigación establecidos:

- Respecto al aprendizaje que han experimentado las estudiantes sobre la planificación didáctica, se puede indicar que han aprendido qué es una planificación abierta y flexible. Conocían, fruto de sus experiencias previas de prácticas, unas programaciones cerradas propias de las unidades didácticas, y han avanzado hacia un modelo de planificación abierta, que integra los intereses de los niños y las niñas y permite incluir en el proceso educativo las situaciones imprevistas. Las maestras indican que han observado una evolución evidente en las estudiantes universitarias a lo largo de los dos meses de prácticas, transitando desde actitudes directivas a otras de mayor sensibilidad y respeto hacia las propuestas de los pequeños.

- El alumnado de la Facultad de Ciencias de la Educación ha aprendido el valor de la gestión colectiva del conocimiento que se produce en las aulas que trabajan a través del ABP. Las maestras opinan que es una de las fortalezas fundamentales de esta metodología y muestran una fuerte adhesión a la concepción del aprendizaje en el marco de la teoría sociocultural, al considerar que el desarrollo personal es una construcción cultural que se lleva a cabo a través de la interacción con otras personas mediante la realización de actividades sociales compartidas. Las estudiantes en su mayoría valoran la estructura cooperativa que existe en las aulas que trabajan ABP, aunque un 30 $\%$ de estudiantes no aprecian los beneficios de dicha estructura.

- Las estudiantes muestran un concepto de diversidad más sesgado y reduccionista que las maestras. Mientras que las primeras identifican la diversidad con la atención a los niños y las niñas discapacitadas, las docentes proyectan una concepción de diversidad amplia, que se puede identificar con las diferencias y singularidades de cada niño o niña. Por ello, ofrecen estrategias didácticas de atención a las diferencias dentro del aula a través de un currículo abierto, flexible, globalizado y versátil.

- Los aprendizajes adquiridos por las estudiantes respecto a la participación de la comunidad en el desarrollo de ABP sugieren que, en su mayoría, las alumnas comprenden que el aprendizaje en la era digital se produce por la conectividad de redes de personas y nodos informacionales. Asumen que los niños y las niñas, que son nativos digitales interactivos, tienen acceso a un proceso de aprendizaje permanente, en el que cada vez son más difusas las fronteras entre el aprendizaje formal e informal. El avance tecnológico democratiza el acceso al conocimiento y diversifica los agentes educativos que lo producen, constatando que a los docentes cada vez les salen más competidores que rivalizan en su misión de transformar la información en conocimiento (Pérez Gómez, 2012).

- La participación de las familias en el ABP es imprescindible para las docentes. Las estudiantes, por su parte, han aprendido que, aun siendo imprescindible la colaboración familiar, no siempre dicha colaboración se realiza de una forma adecuada. Muestran resistencias y reticencias ante determinados comportamientos familiares que consideran inadecuados e intrusivos.

- Uno de los aspectos más valorados por docentes y alumnas, al participar en la experiencia de innovación del Prácticum RIECU, es el proceso de reflexión compartida que semanalmente ponen en marcha las estudiantes y las 
maestras con el fin de analizar situaciones, identificar fortalezas y debilidades, valorar los roles asumidos y proponer materiales y ambientes de aprendizaje para mejorar el ABP. Consideran la pareja educativa como una herramienta fundamental para mejorar la formación docente inicial y continua, y por ello proponen la continuidad de esta innovación.

\section{REFERENCIAS}

Area, M. (2016). Ser docente en la escuela digital. Suplemento Profesional de Magisterio, 22, 15-16. Recuperado de https://goo.gl/SGGsoO.

Balongo, E. y Mérida, R. (2017). Incluir la diversidad familiar en las aulas infantiles a través de los proyectos de trabajo. Profesorado: Revista de Currículum y formación del Profesorado, 21(2), 1-21.

Bates, T. (2015). Teaching in a Digital Age. Recuperado de http://goo.gl/NAvukU

Bauman, Z. (2008). Los retos de la educación en la modernidad líquida. Barcelona: Gedisa.

Busquet-Duran, J., Medina-Cambrón, A. y Ballano-Macías, S. (2013). El uso de las TRIC y el choque cultural en la escuela. Encuentros y desencuentros entre maestros y alumnos. Revista Mediterránea de Comunicación, 4(2), 115-135. http://doi.org/10.14198/MEDCOM2013.4.2.06

Cabello, M. J. (2011). La globalización en Educación Infantil: una forma de acercarse a la realidad. Pedagogía Magna, 11, 189-195.

Capraro, M. M., Capraro, R. M. y Helfeldt, J. (2010). Do differing types of field experiences make a difference in teacher candidates' perceived level of competence? Teacher Education Quarterly, 37(1), 131-154. https://www.jstor.org/stable/23479302

Castells, M. (2001). Internet y la sociedad red. Madrid: La factoría de Ideas.

Castells, M. (Ed.) (2006). La sociedad red: una visión global. Madrid: Alianza Editorial.

Cisterna, F. (2005). Categorización y triangulación como procesos de validación del conocimiento en investigación cualitativa. Theoria, 14(1), 61-71.

Crowther, F., Ferguson, M. y Hann, L. (2009). Developing teacher leaders: How teacher leadership enhances school success. Newbury Park, CA: Corwin Press.

Day, C., Kington, A., Stobart, G. y Sammons, P. (2007). The personal and professional selves of teachers: stable and unstable identities. British Educational Research Journal, 32(4), 601-616. https://doi.org/10.1080/01411920600775316

De Moya, M. V. y Madrid, D. (2015). La Educación Infantil que queremos: investigaciones y experiencias. Ensayos, Revista de la Facultad de Educación de Albacete, 30(2). https://doi.org/10.18239/ensayos.v30i2.934 
Escribano, A. y Martínez, A. (2013). Inclusión educativa y profesorado inclusivo. Aprender juntos para aprender a vivir juntos. Madrid: Narcea.

Gardner, H. (2008). Inteligencias múltiples. La teoría en la práctica. Barcelona: Paidós.

Gardner, H. (2010). La inteligencia reformulada: las inteligencias múltiples en el siglo $X X I$. Barcelona: Paidós.

Gómez, J. L. y Pérez, M. (2011). Bases psicopedagógicas de un modelo de enseñanza-aprendizaje socioconstructivista para entornos virtuales. Indivisa: Boletín de Estudios e Investigación, 12, 61-97.

Hughes, M., Hughes, P. y Hodgkinson, I. R. (2016). In pursuit of a 'whole-brain' approach to undergraduate teaching: implications of the Herrmann brain dominance model. Studies in Higher Education, 42(12), 1-17. http://doi.org/10.1080/03075079.2016.1152463

Imaz, J. I. (2015). Aprendizaje Basado en Proyectos en los grados de Pedagogía y Educación Social: ¿Cómo ha cambiado tu ciudad? Revista Complutense de Educación, 26(3), 679-696. https://doi.org/10.5209/rev RCED.2015.v26.n3.44665

INTEF (2014). Marco común de competencia digital docente. Recuperado de www.slideshare.net/educacionlab/borrador-marcocdd-v1

Korthagen, F. A. J. (2010). La práctica, la teoría y la persona en la formación del profesorado. Revista Interuniversitaria de Formación del Profesorado, 68, 83101.

Mérida, R., González, E., Olivares, M. A. (2012). Redes de aprendizaje escuelas infantiles-universidad. Evaluación de una experiencia de innovación. Cultura y Educación, 24(1), 95-110. https://doi.org/10.1174/113564012799740849

Mérida, R., González, M. E., Olivares, M. A. y Rodríguez, J. (2018). La red de infantil RIECU (Escuela-Centro de profesorado-Universidad) como contexto de aprendizaje para forjar nuevas identidades profesionales docentes en los estudiantes de Grado de Infantil a través del método de proyectos de trabajo. Revista de Innovación y Buenas Prácticas Docentes, 5, 41-45. https://doi.org/10.21071/ripadoc.v5i.10969

Mora, M. (2005). El educador como agente reflexivo en el proceso de formación educativa. Horizontes Educacionales, 10, 71-76.

Olmedo, E. M. (2013). Enfoques de aprendizaje de los estudiantes y metodología docente: Evolución hacia el nuevo sistema de formación e interacción propuesta en el EEES. Revista de Investigación Educativa, 31(2), 411-429. https://doi.org/10.6018/rie.31.2.133501

Pegalajar, M. C. (2018). Formación en competencias en alumnado universitario mediante prácticas basadas en aprendizaje cooperativo. Revista Complutense de Educación, 29(3), 829-845. https://doi.org/10.5209/RCED.53970

Pérez Gómez, A. I. (2012). Educarse en la era digital. Madrid: Morata.

Pérez Gómez, A. I. (2017). Pedagogías para tiempos de perplejidad. De la información a la sabiduría. Rosario, Argentina: Homo Sapiens Ediciones. 
Perrenoud, P. (2007). Diez nuevas competencias para enseñar. México: Editorial Graó.

Prensky, M. (2011). Enseñar a nativos digitales. Madrid: Editorial SM.

Ruiz, M. C. y Mérida, R. (2016). Promover la inclusión de las familias a través del desarrollo de Proyectos de Trabajo. Un estudio de caso. Revista Complutense de Educación, 27(3), 943-961. https://doi.org/10.5209/rev RCED.2016.v27.n3.47022

Sánchez, J., Pérez, F. J. y Venegas, A. J. (2013). Educación emocional en el proceso de formación docente. E-motion: Revista de Educación, Motricidad e Investigación, 1, 184-190.

Vygotsky, L. S. (1989). El desarrollo de los procesos psicológicos superiores. Barcelona: Crítica.

Viñals, A. y Cuenca, J. (2016). El rol del docente en la era digital. Revista Interuniversitaria de Formación del Profesorado, 30(2), 103-114. https://www.redalyc.org/pdf/274/27447325008.pdf

Wake, J. D., Dysthe, O. y Mjelstad, S. (2007). New and Changing Teacher Roles in Higher Education in a Digital Age. Educational Technology \& Society, 10(1), 4051. https://www.jstor.org/stable/jeductechsoci.10.1.40

Zapata, M. (2015). Teorías y modelos sobre el aprendizaje en entornos conectados y ubicuos. Bases para un nuevo modelo teórico a partir de una visión crítica del 'conectivismo'. Education in the knowledge society, 16(1), 69-102. http://dx.doi.org/10.14201/eks201516169102 